\title{
VALORACION DE LA CAPACIDAD SECRETORA DE ACTH POR LA PITUITARIA CON METOPIRONA EN PACIENTES CON AMENORREA
}

\author{
Dr. Fernando del Corral, M.D.* \\ Dr. Carlos A. Salcedo, M.D.* \\ Dr. Carlos E. Niño, M.D.*
}

El manejo de la amenorrea en Ginecología, depende lógicamente de un diagnóstico adecuado. En algunas oportunidades la etiología es obvia o de fácil diagnóstico. En otros casos por el contrario y especialmente en aquellos en que la causa puede ser debida a una alteración del eje hipotálamo-hipófisis, se hace más difícil sobretodo en la diferenciación de las lesiones orgánicas de los funcionales.

Para la valoración de la amenorrea de origen pituitario, nos hemos basado en su clasificación de acuerdo a la determinación de hormonas gonadotropas eliminadas y extraídas de la orina mediante el método de concentración de Bradbury (1) y de bioensayo de Klinefelter (2).

Estas determinaciones nos permiten a grandes rasgos diferenciar tres tipos de amenorrea a saber: hipergonadotrópica, eugonatrópica e hipogonadotrópica. La primera no ofrece ningún problema etiológico, debido a que solo se presenta en casos de agenesia o disgenesia gonadal, en sín-

\footnotetext{
* Departamento de Obstetricia y Ginecología, Facultad de Medicina - Universidad del Valle. Cali - Valle, Colombia, S. A.
}

dromes de castración médica o quirúrgica o en casos de menopausia precoz. En los casos de gonadotropinas normales, asociadas con amenorrea el diagnóstico es muy difícil, el cual generalmente se hace por exclusión y muchas veces solo se llega a él después de diferentes pruebas terapéuticas. En los casos de gonadotropinas bajas o ausentes (hipogonadotrópicas) es imperativo determinar si esta falla se debe a un compromiso tumoral de la glándula $\circ$ a una destrucción glandular como sería el síndrome de Sheehan o por el contrario si se tratara de una alteración pituitaria de tipo funcional.

Una forma indirecta de valorar la capacidad secretora funcional de la pituitaria, fue propuesta por Liddle y col. en 1958 (3).

La metopirona SU4885 Ciba es un inhibidor de la 11 beta hidroxilasa que al ser administrada por vía oral o parenteral produce una disminución de la síntesis de cortisol. Cuando la pituitaria es normal, se produce un aumento compensatorio en la secreción de $A C T H$, que se traduce en un aumento en la producción de 11 desoxicortisol, y un aumento transitorio del cortisol (4). 


\section{MATERIAL Y METODOS}

Procedimiento: Se colectan orinas de 24 horas por dos días, asegurando que esta colección se haya realizado en forma adecuada, mediante la determinación en la misma, de la concentración de creatinina. A continuación se realiza el test con metopirona en la forma siguiente: se administra una dosis de 500 mgrs. de metopirona ( 2 metil-1,2 bis ( 3 piridil)-1-propanone) cada 4 horas por 6 dosis ( 8 a. m. a 4 p. m.); se colectan orinas de 24 horas, durante el día de la administración del bloqueador enzimático y al día siguiente. Los 17 hidroxicorticoides se midieron por el método modificado de Reddy $(6,7,8)$ que se expresan en mgrs./24 horas de orina siendo lo normal para este método una cifra de 2 a 10 mgrs.

A continuación se realiza el test standard de estimulación adrenal con ACTH. Los individuos normales en los cuales no hay alteración hipofisiaria o adrenal, responden con aumento de 2 a 4 veces sobre las cifras basales los 17 hidroxicorticoides, particularmente al día siguiente de administrado el bloqueador enzimático, figura 1. En la misma gráfica se puede apreciar, la talla de respuesta

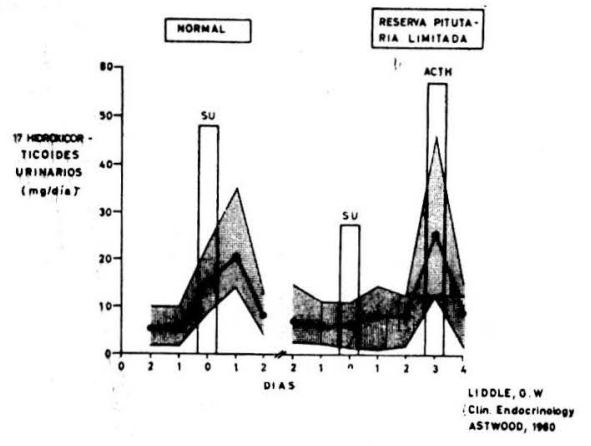

al agente bloqueador en presencia de una "reserva pituitaria limitada".

Con estas premisas hemos practicado la prueba en 25 pacientes con amenorrea para ver las relaciones que pudeiran existir entre la producción gonadotrópica de la hipófisis, con el test de reserva pituitaria con metopirona. Se utilizaron pacientes con amenorrea de por lo menos seis meses de duración, las cuales fueron estudiadas completamente desde el punto de vista endocrinológico.

Los resultados se expresaron en la forma siguiente: el promedio de eliminación de 17 hidroxicorticoides en dos colecciones de orina de 24 horas en condiciones basales, comparadas con la eliminación urinaria en 24 horas de 17 hidroxicorticoides, cifras obtenidas a partir del segundo día de colección después de administrada la metopirona. Los resultados se expresan en miligramos en la siguiente forma: aumento de menos del doble TEST DE RESERVA NEGATIVO: aumento del doble o más TEST DE RESERVA POSITIVO.

Las pacientes se agruparon en la siguiente forma:

Casos

Amenorrea hipogonadotrópica

primaria y secundaria . . . . . 17

Amenorrea primaria y secun-

daria eugonadotrópica . . . . . 6

Amenorrea primaria y secun-

daria hipergonadotrópica . . . 2

La distribución discriminada de ellas puede apreciarse en el Cuadro 1. En el grupo de amenorrea hipogonadotrópica el resultado del test fue positivo en 8 casos y negativo en 9. Cuadro № 2. En los casos negativos, el test de estimulación adrenal con ACTH mostró que ésta era capaz de responder normalmente en 7 casos, incluyendo: Un síndrome de Sheehan, 
CUADRO NN 1

\begin{tabular}{|c|c|c|c|}
\hline & & Casos & Total \\
\hline AMENORREA & PRIMARIA & 3 & 17 \\
\hline $\begin{array}{l}\text { HIPOGONADOTROPICA } \\
(-6.6 \text { UR) }\end{array}$ & & 14 & \\
\hline $\begin{array}{l}\text { AMENORREA } \\
\text { EUGONADOTROPICA }\end{array}$ & PRIMARIA & 0 & \\
\hline (6.6 a 52.8 UR) & SECUNDARIA & 6 & 6 \\
\hline \multirow{3}{*}{$\begin{array}{l}\text { AMENORREA } \\
\text { HIPERGONADOTROPICA } \\
(52.8 \text { UR })\end{array}$} & PRIMARIA & 2 & \\
\hline & SECUNDARIA & 0 & 2 \\
\hline & GRAN TOTAL & & 25 \\
\hline
\end{tabular}

\section{CUADRO № 2}

\begin{tabular}{llr}
\hline & SU & Casos \\
AMENORREA & POSITIVO & 8 \\
HIPOGONADOTROPICA & NEGATIVO & 9 \\
& TOTAL & 17 \\
& S. DE SHEEHAN & 1 \\
NEGATIVOS: SU & PAN HIPOPITUITARISMO & 1 \\
(ACTH RESPUESTA NORMAL) & AMENORREA DEL POST-PARTO & 1 \\
& S. ADRENO-GENITAL LIMITE & 1 \\
& HIPERPLASIA ADRENAL & 1 \\
& AMENORREA HIPOTALAMICA & 2 \\
NEGATIVOS: SU & TOTAL & 7 \\
(ACTH RESPUESTA LIMITADA) & S. DE SHEEHAN & 1 \\
& S. ADRENO-GENITAL LIMITE & 1 \\
& TOTAL & -1 \\
\hline
\end{tabular}

un panhipopituitarismo, una amenorrea del post-parto, un síndrome adreno-genital límite y un caso de hiperplasia adrenal; en dos casos en los cuales el test con SU4885 fué negativo se trataba de un síndrome de Sheehan y un sindrome adreno-genital respectivamente, en los cuales el test de ACTH demostró la presencia de una reserva adrenal limitada. Estos resultados excluyendo los dos últimos, nos demuestran la falta de correlación entre el test de SU4885 y la amenorrea hipogonadotrópica.
En los seis casos de amenorrea eugonadotrópica, la prueba del SU4885 fué positiva en 4 casos y negativa en 2. Cuadro № 3. En los casos en que la respuesta fué positiva se incluye, un caso de Stein-Leventhal, un síndrome adreno-genital por hiperplasia, un síndrome adreno-genital límite y un caso de amenorrea hipotalámica. La prueba fue negativa en dos casos, curiosamente ambos con diagnóstico de Stein-Leventhal, pero con reserva adrenal normal. En los casos de amenorrea hipergonadotrópica el 
CUADRO N: 3

\begin{tabular}{llc}
\hline AMENORREA & SU & Casos \\
EUGONADOTROPICA & POSITIVO & 4 \\
& NEGATIVO & $\frac{2}{6}$ \\
NEGATIVA SU & TOTAL & 2 \\
(ACTH RESPUESTA NORMAL) & S. DE STEIN LEVENTHAL. \\
AMENORREA & POSITIVO & 2 \\
HIPERGONADOTROPICA & & \\
\hline
\end{tabular}

test de SU fue positivo en los dos casos estudiados con diagnóstico de disgenesis gonadal.

\section{COMENTARIO}

La metopirona como test, debe ser considerado actualmente como el más sensitivo disponible para medir la capacidad secretora de ACTH por la pituitaria; existen otras pruebas también de algún valor como serían: 1) la prueba del stress, tal como la hipoglicemia inducida por la insulina, que estimula la secreción de AC$\mathrm{TH}$ probablemente por liberación hipotalámica de un factor "liberador de corticotropina" (9) y 2) una prueba consistente en la capacidad para excretar una sobrecarga de agua; la diuresis normal requiere una concentración normal de cortisol en la circulación que actúe directamente sobre los túbulos renales, la respuesta a la sobrecarga de agua no es una medida directa de reserva de $\mathrm{ACTH}$, pero refleja la presencia de suficiente secreción de ACTH para mantener una normal función adrenal de base, excepción hecha de cuando la función renal es autónoma (10).

Desde 1959 el test de metopirona se ha utilizado extensamente, no obstante los resultados han suscitado mucha discusión en cuanto a la manera de ser interpretados. Para algunos como Kaplan (4) las cifras normales serían desde un aumento escaso hasta cinco veces las cifras basales, para otros (11) los resultados dependen de la dosis administrada y anotan que 500 mgrs. cada 4 horas son inadecuados para un test de reserva, sugiriendo en cambio dosis de 250 mgrs. cada 2 horas.

La vía de administración parece también tener importancia en cuanto a la interpretación de los resultados, debido a que con la metopirona oral, la respuesta obtenida es mayor que cuando se aplica por vía endovenosa (4). La determinación de corticoides urinarios y plasmáticos también establece una diferencia, debido a que la medida plasmática de 11 desoxicorticoides antes y después de la metopirona parece ser la única que puede establecer una diferencia entre las respuestas normales de las anormales. Kaplan sugiere que una respuesta normal sería de por lo menos 7 mgrs. por día en aumento de los 11 desoxicorticoides (4).

$\mathrm{Si}$ comparamos las cifras normales de la respuesta positiva de los casos publicados por Liddle como normales (3) con los obtenidos por nosotros en los 25 casos de amenorrea, a pesar de que la determinación urinaria se hizo por un diferente método, que puede ser comparable, se observa lo siguiente: con un aumento en 25 casos el test fue positivo en 14 que expresados en gráficas y toman- 


\section{CUADRO N: 1}

ESTADO CIVIL - EDAD Y VIVIENDA DISCRIMINADO POR CLASES SOCIALES

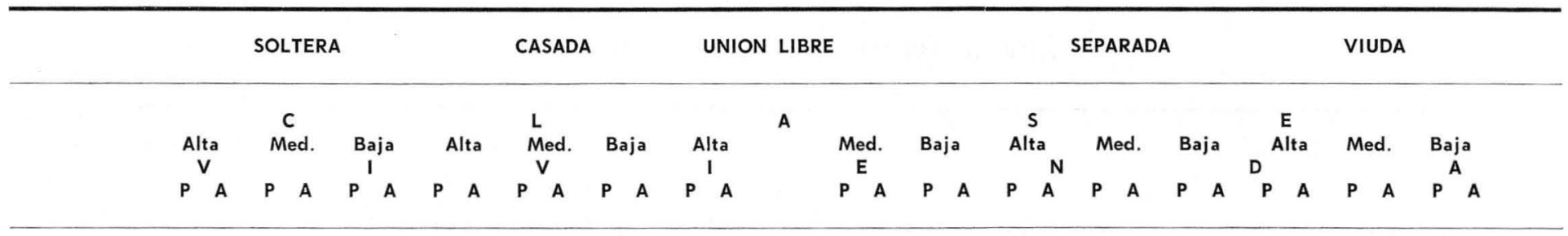

\section{Edad}

$15-20$ a.

$20-24$ a.

$25-29$ a.

$30-34$ a.

$35-39$ a.

$40-44$ a.

$45-49$ a.

$\begin{array}{rrrrrrrr}1 & 2 & & 1 & 1 & & 1 & 1 \\ & & 1 & & & 2 & 3 & 7 \\ 1 & & 1 & 1 & 2 & 5 & 18 & 9 \\ & & 3 & 2 & 5 & & 12 & 6 \\ & & 3 & & 8 & 1 & 5 & 8 \\ & & 2 & & 3 & 1 & 3 & \\ & & & & 3 & & 3 & \end{array}$

\begin{tabular}{|c|c|c|c|c|c|c|c|c|c|c|c|c|c|c|c|}
\hline Total & Clase & 0 & 0 & 4 & 14 & 31 & 76 & 0 & & 17 & 0 & 0 & 0 & 0 & 1 \\
\hline Total & estado $M$. & & & 4 & Casados & & 121 & Unión & libre & 17 & Separados & 0 & & Viudos & 1 \\
\hline
\end{tabular}




\section{CUADRO N: 2}

GRADO DE INSTRUCCION Y RELIGION POR CLASES

\begin{tabular}{|c|c|c|c|c|c|c|c|c|c|c|c|c|}
\hline \multirow[b]{3}{*}{ Grad. Inst. } & \multirow[b]{3}{*}{ Alta } & \multicolumn{3}{|c|}{ CLASE SOCIAL } & \multirow[b]{3}{*}{ Baja } & \multirow[b]{3}{*}{$\%$} & \multirow[b]{3}{*}{ Clases } & \multicolumn{2}{|c|}{ CATOLICA } & \multirow{3}{*}{$\begin{array}{c}\mathbf{N}^{0} \\
\text { Com. }\end{array}$} & \multirow[t]{3}{*}{ Judia } & \multirow[t]{3}{*}{ Otras } \\
\hline & & & & & & & & -1 & +1 & & & \\
\hline & & $\%$ & Media & $\%$ & & & & Comun. & Comun. & & & \\
\hline Analf. & 0 & 0 & 0 & & 18 & 18.3 & Baja & 25 & 65 & 8 & 0 & 0 \\
\hline Prim. & 0 & 0 & 11 & 35.5 & 72 & 73.4 & Media & 7 & 19 & 5 & & \\
\hline Bach. & 11 & 78.5 & 14 & 46.2 & 7 & 7.2 & Alta & & 11 & 3 & & 0 \\
\hline Comerc. & 2 & 14.2 & 5 & 16 & 1 & 11 & & & & & & \\
\hline Univers. & 0 & 0 & 0 & & 0 & 0 & Total & 32 & 95 & 16 & & \\
\hline Otros & 1 & 7.3 & 1 & 3.2 & 0 & 0 & $\%$ & 22.37 & 66.43 & 12.10 & & \\
\hline TOTAL & 14 & & 31 & & 98 & & - & & & & & \\
\hline
\end{tabular}


do los promedios y comparándolos, observamos que estas cifras son notoriamente más bajas en los casos de amenorrea, figura 2. Parece por

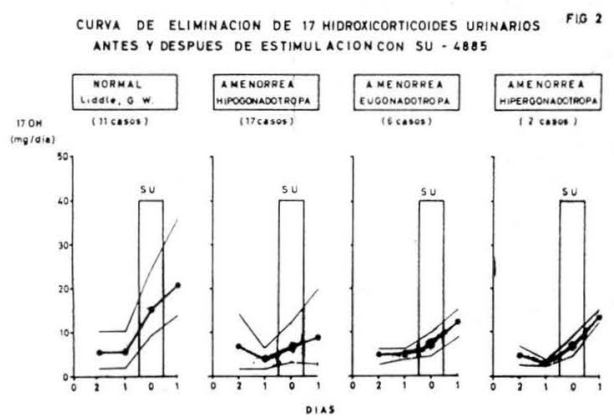

los resultados obtenidos, que la metopirona es un test específico para ACTH, que no es específico en pacientes con hipotiroidismo y que en los 25 casos estudiados por nosotros, no parece existir ninguna relación entre el test de metopirona y la eliminación gonadotrópica, figura 3.

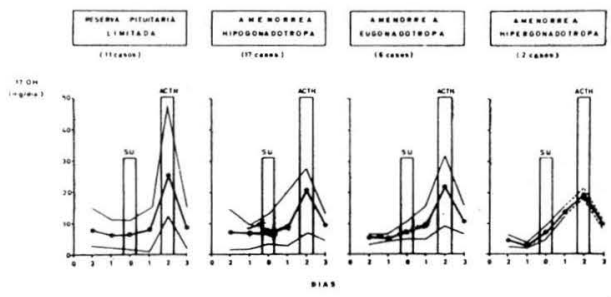

Actualmente estamos determinando las curvas normales promedio en individuos normales para poder comparar en una forma adecuada los resultados en pacientes con problemas endocrinos.

El manejo de la amenorrea, continúa siendo un problema etiológico particularmente en los casos en que las gonadotropinas se encuentran normales o bajas. Estamos iniciando un estudio para determinar, si el test de reserva pituitaria por metopirona, pudiera tener alguna relación, con la respuesta terapéutica de la ovulación inducida con "clomid" en pacientes con amenorrea y su posible relación entre los tests con SU positivos o negativos.

\section{RESUMEN}

Se presenta el test de reserva pituitaria con Metopirona (SU4885) en 25 pacientes con amenorrea de diversos tipos, correlacionando las respuestas positivas y negativas con la eliminación de gonadotropinas urinarias. No parece existir ninguna relación entre el test de reserva pituitaria y los diversos tipos de amenorrea, de acuerdo con la eliminación de gonadotropinas urinarias.

\section{BIBLIOGRAFIA}

1 BRADBURY: Proc. Soc. Exper. Biol. Am. $71: 228,1949$.

2 KLINEFELTER-ALBRIGHT, J.: Clin. Endo. $3: 529,1943$.

3 LIDDLE, G. W.; D'ISLAND, E. M. LANCE AND A. P. HARRIS: J. Clin. Endocrinol \& Metab. 18:906, 1958.

4 KAPLAN, N.: Assesment of pituitary ACTH secretory capacity with Metopirone: 1 Interpretation. J. of Clin. Endocrinol \& Metab. 23, 9, 945:952 Sept. 1963.

5 LIDDLE, G. N.: Test of Adrenocortical reserve, pituitary reserve and pituitary-adrenal suppressibility. Clinical Endocrinology I. Astwood. E. B. Editor. Grune \& Stratton. New York 1960.

6 REDDY, W. J.: Modification of the ReddyJenkins-Thorn method for the stimation of 17-hydroxicorticoids in urine, Metabolism $3: 489,1954$

7 REDDY, W. J.; JENKINS, D. AND THORN, G. W.: Estimation of 17-hydroxycorticoids in urine, Metabolism 1:511, 1952.

8 REDDY, W. J. et al.: Determination of total 17 hydroxycorticosteroids in plasma, J. of $\mathrm{Cl}$. Endocrinol. \& Bet. 16:380, 1956.

9 SAYERS, G.; AND P. C. ROYCE: In Astwood Grune \& Stratton, New York p. 323, 1960.

10 KLEEMAN, C. R.; H. M. MAXWELL AND R. E. ROCKNEY: J. Clin. Invest. 37:1799, 1958.

11 BUSS, O. C. BINDER AND PETERSEN. Lancet $1: 1040,1962$. 